Cahiers $d u$ MONDE RUSSE

\section{Cahiers du monde russe}

Russie - Empire russe - Union soviétique et États indépendants

$53 / 4 \mid 2012$

Varia

\title{
Daniel' Bovua [Daniel Beauvois], Gordiev Uzel Rossijskoj imperii
}

Aleksej Miller

\section{OpenEdition}

\section{Journals}

Édition électronique

URL : http://journals.openedition.org/monderusse/7732

DOI : 10.4000/monderusse.7732

ISSN : $1777-5388$

\section{Éditeur}

Éditions de l'EHESS

\section{Édition imprimée}

Date de publication : 15 décembre 2012

ISSN : $1252-6576$

Référence électronique

Aleksej Miller, « Daniel' Bovua [Daniel Beauvois], Gordiev Uzel Rossijskoj imperii », Cahiers du monde russe [Онлайн], 53/4 | 2012, Выложить онлайн 11 avril 2013, Наводить справки в 25 septembre 2020. URL : http://journals.openedition.org/monderusse/7732 ; DOI : https://doi.org/10.4000/ monderusse. 7732

Ce document a été généré automatiquement le 25 septembre 2020.

(c) École des hautes études en sciences sociales 


\title{
Daniel' Bovua [Daniel Beauvois], Gordiev Uzel Rossijskoj imperii
}

\author{
Aleksej Miller
}

\section{RÉFÉRENCE}

Daniel' BovuA [Daniel Beauvois], Gordiev Uzel Rossijskoj imperii. Vlast', sljahta i

narod na Pravoberežnoj Ukraine (1793-1914) [Le nœud gordien de l'Empire russe : le pouvoir, la noblesse polonaise et le peuple en Ukraine de la rive droite (1793-1914)]. Moscou : Novoe literaturnoe obozrenie, 2011, 997 p. (trad. du français par M. Krisan')

1 ВСЮ СВОЮ НАУЧНУЮ КАРЬЕРУ ДАНИЭЛЬ БОВУА ПОСВЯТИЛ ИЗУЧЕНИЮ ИСТОРИИ ЗАПАДНЫХ ОКРАИН РОССИЙСКОЙ ИМПЕРИИ. ОГРОМНАЯ ПО ОБЪЕМУ (1000 СТРАНИЦ МЕЛКИМ ШРИФТОМ) КНИГА «ГОРДИЕВ УЗЕЛ РОССИЙСКОЙ ИМПЕРИИ: ВЛАСТЬ, ШЛЯХТА И НАРОД НА ПРАВОБЕРЕЖНОЙ УКРАИНЕ (1793-1914)» (МОСКВА, НЛО, 2011) - ВО МНОГОМ СУММИРУЕТ ЭТИ ИССЛЕДОВАНИЯ. НАЧИНАЛ БОВУА КАК РУСИСТ, ТАК ЧТО, ДУМАЮ, ДЛЯ НЕГО ИЗДАНИЕ ПО-РУССКИ ЕГО орus magnum - ВАЖНОЕ СОБЫТИЕ, СВОЕГО РОДА «ИСТОРИЧЕСКАЯ СПРАВЕДЛИВОСТЬ», ВЕДЬ ПРЕЖДЕ ЕГО КНИГИ НА РУССКИЙ НЕ ПЕРЕВОДИЛИСЬ. БЕЗУСЛОВНО, ВАЖНЫМ СОБЫТИЕМ СТАЛА ЭТА КНИГА И ДЛЯ ИСТОРИОГРАФИИ ВОСТОЧНОЙ ЕВРОПЫ, КОТОРАЯ, ПО-ПРЕЖНЕМУ, ВО МНОГОМ ИСПОЛЬЗУЕТ РУССКИЙ ЯЗЫК КАК lingua franca ДАЖЕ В БОЛЬШЕЙ СТЕПЕНИ, ЧЕМ Английский.

2 В ФОКУСЕ КНИГИ - СУДЬБА ПОЛЬСКОЙ ШЛЯХТЫ НА ПРАВОМ БЕРЕГУ ДНЕПРА, ИЛИ В ЮГО-ЗАПАДНОМ КРАЕ РОССИЙСКОЙ ИМПЕРИИ, СО ВРЕМЕН РАЗДЕЛОВ РЕЧИ ПОСПОЛИТОЙ ДО НАЧАЛА ПЕРВОЙ МИРОВОЙ ВОЙНЫ. КНИГА СОСТОИТ ИЗ ТРЕХ ЧАСТЕЙ, КАЖДАЯ ИЗ КОТОРЫХ ОПИРАЕТСЯ НА УЖЕ ИЗВЕСТНЫЕ ФРАНЦУЗСКОМУ ЧИТАТЕЛЮ РАБОТЫ. ПЕРВАЯ ЧАСТЬ, ОХВАТЫВАЮЩАЯ ПЕРИОД С 1793 ПО 1830 ГОДЫ, ОСНОВАНА НА КНИГЕ Pouvoir russe et noblesse polonaise en Ukraine: 1793-1830 (2003). В НЕЙ ПОКАЗАНЫ ИСТОРИЧЕСКИЕ КОРНИ НЕОБЫЧНО ВЫСОКОЙ ЧИСЛЕННОСТИ ПОЛЬСКОЙ ШЛЯХТЫ ПРАВОБЕРЕЖНОЙ УКРАИНЫ, ЕЕ ВНУТРЕННЯЯ НЕОДНОРОДНОСТЬ. ОСОБЫЙ ИНТЕРЕС (И 
НЕСКРЫВАЕМОЕ СОЧУВСТВИЕ) АВТОРА ВЫЗЫВАЕТ ПРЕЖДЕ НЕ ПОЛУЧАВШАЯ ДОЛЖНОГО ВНИМАНИЯ ГРУППА БЕЗЗЕМЕЛЬНОЙ ШЛЯХТЫ. БОВУА ПОДЧЕРКИВАЕТ, ЧТО ЕЕ СТАТУС БЫЛ СПОРНЫМ ЕЩЕ ДО РАЗДЕЛОВ, И ЧТО ВЛАСТИ ИМПЕРИИ ВПЛОТЬ ДО ВОССТАНИЯ 1830 Г. РАССМАТРИВАЛИ ЦЕЛЫЙ РЯД ПРОЕКТОВ, ПРЕДУСМАТРИВАВШИХ ЧАСТИЧНОЕ ДЕКЛАССИРОВАНИЕ ЭТОЙ ГРУППЫ, ЧИСЛЕННОСТЬ КОТОРОЙ (БОЛЕЕ 260 ТЫСЯЧ) БЫЛА СОПОСТАВИМА С ЧИСЛЕННОСТЬЮ ВСЕГО ДВОРЯНСТВА ВЕЛИКОРУССКИХ ГУБЕРНИЙ. АВТОР ТАКЖЕ ПОКАЗЫВАЕТ, КАК В ЭТОТ ПЕРИОД ПОСТЕПЕННО ЗАКРЫВАЛИСЬ ВОЗМОЖНОСТИ БЕЗЗЕМЕЛЬНОЙ ШЛЯХТЫ ПОВЫСИТЬ СВОЙ СОЦИАЛЬНЫЙ СТАТУС ЧЕРЕЗ ОБРАЗОВАНИЕ. ВТОРАЯ ЧАСТЬ ОПИРАЕТСЯ НА КНИГУ Le noble, le serf et le revizor (1985), И РАССКАЗЫВАЕТ О ТОМ, КАК ПОСЛЕ ВОССТАНИЯ, В ПЕРИОД 30-40-Х ГОДОВ ЛИШЬ 1/5 ШЛЯХТЫ РЕГИОНА СМОГЛА СОХРАНИТЬ СВОЙ ДВОРЯНСКИЙ СТАТУС. БОВУА СЧИТАЕТ, ЧТО ЭТО БЫЛ «САМЫЙ МАСШТАБНЫЙ ПРИМЕР СОЦИАЛЬНОГО МАНИПУЛИРОВАНИЯ В ЕВРОПЕ ДО НАЧАЛА ХХ В.», В ХОДЕ КОТОРОГО 370 ТЫС. БЕЗЗЕМЕЛЬНЫХ ШЛЯХТИЧЕЙ БЫЛИ ИСКЛЮЧЕНЫ ИЗ ПРИВИЛЕГИРОВАННОГО СОСЛОВИЯ. АВТОР ОТМЕЧАЕТ, ЧТО СОСЛОВНАЯ СОЛИДАРНОСТЬ БОГАТОЙ ШЛЯХТЫ С ЭТОЙ ГРУППОЙ, ОКАЗАВШЕЙСЯ ПОД УГРОЗОЙ, БЫЛА ОГРАНИЧЕННОЙ. ЗДЕСЬ ЖЕ РАССМАТРИВАЕТСЯ ПРОТИВОСТОЯНИЕ ПОЛЬСКОЙ ШЛЯХТЫ И ИМПЕРИИ В БОРЬБЕ ЗА ИДЕНТИЧНОСТЬ И ЛОЯЛЬНОСТЬ МЕСТНОГО КРЕСТЬЯНСТВА. ТРЕТЬЯ ЧАСТЬ ОСНОВАНА НА КНИГЕ La bataille de la terre en Ukraine, 1863-1914 (1993). В НЕЙ АВТОР СОСРЕДОТОЧЕН, ПРЕЖДЕ ВСЕГО, НА БОРЬБЕ ЗА ЗЕМЛЮ ПОСЛЕ ВОССТАНИЯ 1863 Г., КОГДА ВЛАСТИ ИМПЕРИИ ПОСТАВИЛИ СВОЕЙ ЦЕЛЬЮ ПОДОРВАТЬ ВЛИЯНИЕ ПОЛЬСКОГО КРУПНОГО ЗЕМЛЕВЛАДЕНИЯ В КРАЕ И УСИЛИТЬ ВЛИЯНИЕ РУССКИХ ПОМЕЩИКОВ. ЭТА ПОЛИТИКА ВЛАСТЕЙ ДАЛА ОГРАНИЧЕННЫЕ РЕЗУЛЬТАТЫ - ЕСЛИ В 1860 Г. В РУКАХ ПОЛЯКОВ НАХОДИЛОСЬ 5/6 КРУПНОЙ ЗЕМЕЛЬНОЙ СОБСТВЕННОСТИ, ТО К НАЧАЛУ ПЕРВОЙ МИРОВОЙ ВОЙНЫ ОНИ СОХРАНИЛИ КОНТРОЛЬ НАД ПОЛОВИНОЙ ВСЕХ ЗЕМЕЛЬ КРАЯ. МАТЕРИАЛЬНОЕ БЛАГОСОСТОЯНИЕ КРУПНЫХ ПОМЕЩИКОВ К НАЧАЛУ ХХ В. БЫЛО КАК НИКОГДА ПРОЧНЫМ, ПОСКОЛЬКУ ОНИ СУМЕЛИ УСПЕШНО ПРИСПОСОБИТЬСЯ К КАПИТАЛИСТИЧЕСКОЙ ТРАНСФОРМАЦИИ, И ЧАСТО СОТРУДНИЧАЛИ В ЭТОЙ СФЕРЕ С РУССКИМИ ПОМЕЩИКАМИ. ЭТА ЧАСТЬ ПРОДОЛЖАЕТ РАССКАЗ О ТРАГЕДИИ БЕЗЗЕМЕЛЬНОЙ ШЛЯХТОЙ, КОТОРАЯ В ЭТОТ ПЕРИОД, ПОСЛЕ УТЕРИ СВОЕГО СТАТУСА, СТАЛА ОБЪЕКТОМ МАССОВЫХ ВЫСЕЛЕНИЙ, ЧТО ОТВЕЧАЛО ИНТЕРЕСАМ И АДМИНИСТРАЦИИ, И КРУПНЫХ ПОЛЬСКИХ ЗЕМЛЕВЛАДЕЛЬЦЕВ.

РЕЦЕНЗИРУЕМАЯ КНИГА УЖЕ ВЫХОДИЛА НА ПОЛЬСКОМ ЯЗЫКЕ ${ }^{1}$, НО АВТОР ДОБАВИЛ В РУССКОЕ ИЗДАНИЕ НОВУЮ ГЛАВУ И ВНЕС ДРУГИЕ ИЗМЕНЕНИЯ. НАЗВАНИЕ КНИГИ В РУССКОМ ПЕРЕВОДЕ УДАЧНЕЕ, ЧЕМ В ПОЛЬСКОМ, ГДЕ РЕЧЬ ИДЕТ О «ТРЕУГОЛЬНИКЕ», ПОТОМУ ЧТО ВЗАИМОДЕЙСТВУЮЩИХ СТОРОН В ЭТОЙ ИСТОРИИ МНОГО БОЛЬШЕ, ЧЕМ ТРИ. ВО-ПЕРВЫХ, В ЭТОМ ВЗАИМОДЕЙСТВИИ УЧАСТВОВАЛИ И ПРЕДСТАВИТЕЛИ ДРУГИХ ЭТНИЧЕСКИХ ГРУПП - ПРЕЖДЕ ВСЕГО ЕВРЕИ, А ТАКЖЕ НЕМЕЦКИЕ И ЧЕШСКИЕ КОЛОНИСТЫ, ПОЗДНЕЕ И ВЕЛИКОРУССКИЕ РАБОЧИЕ. БОВУА ОГОВАРИВАЕТСЯ, ЧТО ЭТИ АКТОРЫ ИССЛЕДОВАНЫ ИМ ЛИШЬ КОСВЕННО, НО СООБЩАЕТ ЧИТАТЕЛЮ ВАЖНЫЕ СВЕДЕНИЯ О РОЛИ ЕВРЕЙСКИХ ФАКТОРОВ И АРЕНДАТОРОВ, ЧЕШСКИХ И НЕМЕЦКИХ ПЕРЕСЕЛЕНЦЕВ. ВО-ВТОРЫХ, НЕСМОТРЯ НА ТО, ЧТО В СВОИХ ЭТИЧЕСКИХ СУЖДЕНИЯХ И МОРАЛЬНЫХ ОЦЕНКАХ АВТОР ИНОГДА ОПЕРИРУЕТ УПРОЩАЮЩИМИ ПОНЯТИЯМИ ПОЛЬСКИЙ ПОМЕЩИК, УКРАИНСКИЙ КРЕСТЬЯНИН И РУССКАЯ ИМПЕРСКАЯ ВЛАСТЬ - В СВОЕМ ИСТОРИЧЕСКОМ АНАЛИЗЕ БОВУА ПОЛЬЗУЕТСЯ, КАК ПРАВИЛО, БОЛЕЕ ДРОБНЫМИ И ТОЧНЫМИ КАТЕГОРИЯМИ. ПРЕЖДЕ ВСЕГО, В КНИГЕ ПОКАЗАНА НЕОДНОРОДНОСТЬ 
ШЛЯХТЫ, ПРОТИВОРЕЧИВОСТЬ ИНТЕРЕСОВ БОГАТЫХ ПОЛЬСКИХ ЗЕМЛЕВЛАДЕЛЬЦЕВ И БЕЗЗЕМЕЛЬНЫХ ЧИНШЕВИКОВ.

ЛЮБАЯ ПОПЫТКА ПЕРЕСКАЗАТЬ КНИГУ ТАКОГО ОБЪЕМА В РЕЦЕНЗИИ ЗАРАНЕЕ ОБРЕЧЕНА НА НЕУДАЧУ. ЛИШЬ ОБОЗНАЧИВ ОСНОВНЫЕ ТЕМЫ (А ИМЕННО СУДЬБА ШЛЯХТЫ ЮГО-ЗАПАДНОГО КРАЯ, ЕЕ ПРАВОВОЙ СТАТУС, ОБРАЗОВАТЕЛЬНЫЕ УЧРЕЖДЕНИЯ, ЭКОНОМИЧЕСКАЯ АКТИВНОСТЬ, БОРЬБА ЗА СТАТУС И ЗЕМЛЮ С ИМПЕРСКОЙ АДМИНИСТРАЦИЕЙ), И ОТМЕТИВ, ЧТО ЭТИ СЮЖЕТЫ ОСВЕЩЕНЫ ВЕСЬМА ОСНОВАТЕЛЬНО, ПОГОВОРИМ О НЕДОСТАТКАХ И СПОРНЫХ МОМЕНТАХ. КРОМЕ ТОГО, ВАЖНО ОЦЕНИТЬ, КАКОЕ МЕСТО ЭТА КНИГА ЗАЙМЕТ В РОССИЙСКОМ ИСТОРИОГРАФИЧЕСКОМ ЛАНДШАФТЕ, И, ШИРЕ, В КОНТЕКСТЕ ИСТОРИОГРАФИИ РЕГИОНА.

БОВУА, КАК, СОБСТВЕННО, ЛЮБОМУ ИССЛЕДОВАТЕЛЮ ВЗАИМОДЕЙСТВИЙ СО СТОЛЬ СЛОЖНОЙ СТРУКТУРОЙ, НЕ УДАЕТСЯ С РАВНОЙ ПРИСТАЛЬНОСТЬЮ РАССМОТРЕТЬ ТЕ ТРИ СТОРОНЫ, КОТОРЫЕ УПОМЯНУТЫ В ЗАГОЛОВКЕ ЕГО КНИГИ. В ЦЕНТРЕ ЕГО ВНИМАНИЯ - ШЛЯХТА. МЕНЕЕ ПОДРОБНО РАССМОТРЕНЫ ВЛАСТИ ИМПЕРИИ, ЕЩЕ МЕНЬШЕ ВНИМАНИЯ УДЕЛЕНО МЕСТНОМУ КРЕСТЬЯНСТВУ.

ПОРОЙ БОВУА ВПАДАЕТ В ГРЕХ, ХАРАКТЕРНЫЙ ДЛЯ МНОГИХ ИСТОРИКОВ, СМОТРЯЩИХ НА ИМПЕРИЮ С ЕЕ БЫВШИХ ОКРАИН, НЕ ВИДИТ ВСЕХ ДИЛЕММ И ПРОТИВОРЕЧИЙ ПРАВЯЩИХ ЭЛИТ И ПРЕДСТАВЛЯЕТ ЛОГИКУ ДЕЙСТВИЙ ВЛАСТЕЙ СЛИШКОМ УПРОЩЕННО. ТАК, ОН ОШИБОЧНО СЧИТАЕТ, ЧТО СТРЕМЛЕНИЕ К РУСИФИКАЦИИ ЮГОЗАПАДНОГО КРАЯ ОПРЕДЕЛЯЛО ПОЛИТИКУ ВЛАСТЕЙ В ТЕЧЕНИЕ ВСЕГО ПЕРИОДА ОТ РАЗДЕЛОВ И ДО РАЗВАЛА ИМПЕРИИ. В ЦАРСТВОВАНИЕ АЛЕКСАНДРА І ВЛАСТИ ИМПЕРИИ ПЫТАЛИСЬ ОПЕРЕТЬСЯ НА РЕГИОНАЛЬНЫЕ ЭЛИТЫ, И ЭКСПЕРИМЕНТИРОВАЛИ С РАЗЛИЧНЫМИ ФОРМАМИ АВТОНОМИИ И В ЦАРСТВЕ ПОЛЬСКОМ, И В ВЕЛИКОМ КНЯЖЕСТВЕ ФИНЛЯНДСКОМ, И В БЕССАРАБИИ, НЕ ГОВОРЯ УЖЕ О СУЩЕСТВЕННОЙ СОСЛОВНОЙ АВТОНОМИИ БАЛТИЙСКОГО ДВОРЯНСТВА. ПРИ АНАЛИЗЕ ОБЩЕЙ ТЕНДЕНЦИИ К СОКРАЩЕНИЮ ВЛИЯНИЯ ПЕРИФЕРИЙНЫХ ЭЛИТ, ПОМИМО ПОСТЕПЕННОГО РОСТА ЗНАЧЕНИЯ НАЦИОНАЛИЗМА КАК ЛЕГИТИМИРУЮЩЕГО ПРИНЦИПА, СТОИЛО БЫ ОБРАТИТЬ ВНИМАНИЕ И НА РОСТ БЮРОКРАТИЧЕСКОЙ ОСНАЩЕННОСТИ ИМПЕРИИ И ПЕРЕХОД К ПРЯМЫМ ФОРМАМ ПРАВЛЕНИЯ НА ОКРАИНАХ КАК ЧАСТИ МОДЕРНИЗАЦИОННЫХ ПРЕОБРАЗОВАНИЙ.

7 КОНЕЧНО, ВАЖНУЮ РОЛЬ В ДИНАМИКЕ ЭТИХ ПРОЦЕССОВ СЫГРАЛИ ВОССТАНИЯ 1830-1831 И 1863-1864 ГОДОВ. ИМЕННО ВОССТАНИЯ ЯВЛЯЮТСЯ РУБЕЖАМИ, КОТОРЫЕ ДЕЛЯТ КНИГУ БОВУА НА ТРИ СТРУКТУРНЫЕ ЧАСТИ. ОДНАКО САМИ ВОССТАНИЯ, ЧТО ДОСТОЙНО СОЖАЛЕНИЯ, ПОЧТИ НЕ ОБСУЖДАЮТСЯ, ОНИ КАК БЫ ПРОВАЛИВАЮТСЯ В СТЫКИ МЕЖДУ ЭТИМИ ЧАСТЯМИ КНИГИ ${ }^{2}$ В НЕКОТОРОМ СМЫСЛЕ ЭТО СХОЖЕ С БЫТОВАВШИМ ДО НЕДАВНЕГО ВРЕМЕНИ ОТНОШЕНИЕМ К ИСТОРИИ ПЕРВОЙ МИРОВОЙ ВОЙНЫ - КОГДА ИСТОРИКИ ЗАНИМАЛИСЬ ТЕМ ИЛИ ИНЫМ ПРОЦЕССОМ ДО ВОЙНЫ, ИЛИ ПОСЛЕ НЕЕ. МЕЖДУ ТЕМ, ВО ВРЕМЯ ВОССТАНИЙ, И, ТЕМ БОЛЕЕ, ВЕЛИКОЙ ВОЙНЫ, ПРОИСХОДИЛИ ИЗМЕНЕНИЯ И ВЗАИМОДЕЙСТВИЯ НЕВИДАННОЙ В ОБЫЧНЫХ УСЛОВИЯХ ИНТЕНСИВНОСТИ, ЧТО ДАВАЛО МОЩНУЮ ИНЕРЦИЮ ПОСЛЕДУЮЩИМ СОБЫТИЯМ. ЭТО КАСАЕТСЯ И ВЗАИМООТНОШЕНИЙ КРЕСТЬЯН И ШЛЯХТЫ, И ШЛЯХТЫ С ИМПЕРСКОЙ ВЛАСТЬЮ. МНЕ, НАПРИМЕР, БЫЛО БЫ ОЧЕНЬ ИНТЕРЕСНО УЗНАТЬ МНЕНИЕ БОВУА О ТОМ, ПОЧЕМУ РОССИЙСКИЕ ВЛАСТИ НИКОГДА НЕ ПРИБЕГЛИ К ТАКТИКЕ АВСТРИЙСКИХ ВЛАСТЕЙ, КОТОРЫЕ НЕ СТЕСНЯЛИСЬ ДАТЬ ВОЛЮ КРЕСТЬЯНСКОЙ НЕНАВИСТИ К КРУПНЫМ ЗЕМЛЕВЛАДЕЛЬЦАМ В СЛУЧАЕ НЕЛОЯЛЬНОГО ПОВЕДЕНИЯ ПОСЛЕДНИХ. 
СОБСТВЕННО, КАК ТОЛЬКО ШЛЯХТА ОСТАЛАСЬ С КРЕСТЬЯНАМИ ОДИН НА ОДИН В 1917 Г., ПОЛЬСКОМУ ЗЕМЛЕВЛАДЕНИЮ В КРАЕ ТУТ ЖЕ ПРИШЕЛ КОНЕЦ. В ТО ЖЕ ВРЕМЯ, НУЖНО ПОДЧЕРКНУТЬ, ЧТО ИМЕННО ТАКАЯ АСИММЕТРИЯ, С ФОКУСОМ НА ИСТОРИЮ ШЛЯХТЫ КРАЯ, И ДЕЛАЕТ КНИГУ ОСОБЕННО ЦЕННОЙ В КОНТЕКСТЕ ИСТОРИОГРАФИИ РЕГИОНА, КОТОРАЯ ЗА ПОСЛЕДНЕЕ ДЕСЯТИЛЕТИЕ ПОСВЯТИЛА ЗАПАДНЫМ ОКРАИНАМ ИМПЕРИИ ЦЕЛЫЙ РЯД МОНОГРАФИЙ И КОЛЛЕКТИВНЫХ ТРУДОВ. ДЕЛО В ТОМ, ЧТО У РОССИЙСКИХ АВТОРОВ, ИСПОЛЬЗУЮЩИХ СИТУАЦИОННЫЙ ПОДХОД, ТО ЕСТЬ СТРЕМЯЩИХСЯ РАССМАТРИВАТЬ ВЗАИМОДЕЙСТВИЯ РАЗЛИЧНЫХ ПОЛИТИЧЕСКИХ АКТЕРОВ И СОЦИАЛЬНЫХ ГРУПП, БОЛЬШЕ ВНИМАНИЯ УДЕЛЕНО АНАЛИЗУ ПОЛИТИКИ ВЛАСТЕЙ ИМПЕРИИ. УКРАИНСКИЕ АВТОРЫ БОЛЬШЕ ВНИМАНИЯ УДЕЛАЮТ, КОНЕЧНО, НАЦИОНАЛЬНОМУ ДВИЖЕНИЮ. ИМЕННО ШЛЯХТА, БУДУЧИ ОДНИМ ИЗ КЛЮЧЕВЫХ АКТОРОВ, ЧАСТО ОСТАВАЛАСЬ БЕЗ ДОЛЖНОГО ВНИМАНИЯ. БОВУА ЗАКРЫВАЕТ ЭТУ ВАЖНУЮ ЛАКУНУ.

ПОТЕНЦИАЛЬНО ЭТА КНИГА ОЧЕНЬ ВАЖНА ДЛЯ УКРАИНСКОЙ ИСТОРИОГРАФИИ. ДЕЛО В ТОМ, ЧТО СЕГОДНЯ ИМЕННО УКРАИНСКИЕ ИСТОРИКИ В ОПОРЕ НА МЕСТНЫЕ АРХИВЫ КОЛЛЕКТИВНЫМИ УСИЛИЯМИ МОГЛИ БЫ СДЕЛАТЬ МНОГОЕ ДЛЯ ИЗУЧЕНИЯ ИСТОРИИ ШЛЯХТЫ, И ОСОБЕННО ТОЙ МНОГОЧИСЛЕННОЙ ДЕКЛАССИРОВАННОЙ ШЛЯХТЫ, О КОТОРОЙ С ТАКИМ СОЧУВСТВИЕМ ГОВОРИТ В СВОЕЙ КНИГЕ БОВУА 3 ВОЗМОЖНО, ЕСТЬ ИСТОЧНИКИ, КОТОРЫЕ ПОЗВОЛЯТ ПОКАЗАТЬ ЭТУ ГРУППУ ИЛИ ОТДЕЛЬНЫХ ЕЕ ПРЕДСТАВИТЕЛЕЙ БОЛЕЕ ОБЪЕМНО. К СОЖАЛЕНИЮ, В КНИГЕ МЫ ПОЧТИ НЕ ВСТРЕЧАЕМ ВЫДЕРЖЕК ИЗ ДНЕВНИКОВ, ПИСЕМ, МЕМУАРОВ, В КОТОРЫХ МОЖНО БЫЛО БЫ УСЛЫШАТЬ ГОЛОС ШЛЯХТЫ. ТА МЕЛКАЯ БЕЗЗЕМЕЛЬНАЯ ШЛЯХТА, СУДЬБЕ КОТОРОЙ УДЕЛЯЕТ СТОЛЬКО ВНИМАНИЯ АВТОР, ОСТАЕТСЯ ПРАКТИЧЕСКИ БЕЗГОЛОСОЙ И БЕЗЛИКОЙ. НАПРИМЕР, БЫЛО БЫ ОЧЕНЬ ИНТЕРЕСНО УЗНАТЬ, КАК БЕЗЗЕМЕЛЬНАЯ ШЛЯХТА ОТНОСИЛАСЬ К КРЕСТЬЯНАМ. МОЖНО ПРЕДПОЛОЖИТЬ, ЧТО У МНОГИХ ЭТО ОТНОШЕНИЕ НЕ БЫЛО ЛИШЕНО СОСЛОВНОЙ СПЕСИ, ПОДЧЕРКИВАНИЯ ИНОГО, БЛАГОРОДНОГО ПРОИСХОЖДЕНИЯ. ЧЕМ БЛИЖЕ ПО РЕАЛЬНЫМ СОЦИАЛЬНЫМ УСЛОВИЯМ СТОЯЛА БЕЗЗЕМЕЛЬНАЯ ШЛЯХТА К КРЕСТЬЯНАМ, ТЕМ СИЛЬНЕЕ ОНА ДОЛЖНА БЫЛА ПОДЧЕРКИВАТЬ СВОЮ ИНАКОВОСТЬ. МЫ ЗНАЕМ ТАКЖЕ НЕМНОГОЧИСЛЕННЫЕ СЛУЧАИ ХЛОПОМАНСТВА», КОГДА ВЫХОДЦЫ ИЗ ШЛЯХЕТСКИХ СЕМЕЙ НАЧИНАЛИ ИДЕНТИФИЦИРОВАТЬ СЕБЯ С ИНТЕРЕСАМИ МЕСТНОГО КРЕСТЬЯНСТВА, ВПЛОТЬ ДО ОТКАЗА ОТ ПОЛЬСКОСТИ. НО ВСТРЕЧАЛИСЬ И ЕЩЕ БОЛЕЕ НЕОЖИДАННЫЕ ТРАЕКТОРИИ. В СВОИХ ИССЛЕДОВАНИЯХ МНЕ ПРИШЛОСЬ СТОЛКНУТЬСЯ С ВЫХОДЦЕМ ИЗ БЕДНОЙ ВОЛЫНСКОЙ ШЛЯХТЫ ОРЕСТОМ НОВИЦКИМ, КОТОРЫЙ НАЧАЛ СВОЮ КАРЬЕРУ В КИЕВЕ КАК ПРОФЕССОР ПОЛЬСКОГО ЯЗЫКА В УНИВЕРСИТЕТЕ. ВСКОРЕ ОН СТАЛ ПРОФЕССОРОМ ФИЛОСОФИИ И ЦЕНЗОРОМ. ИМЕННО В ЭТОЙ ПОСЛЕДНЕЙ РОЛИ ОН ВЫСТУПИЛ ИНИЦИАТОРОМ ЗАПРЕТА НА ЛАТИНИЦУ ДЛЯ «МАЛОРУССКОГО НАРЕЧИЯ» В 1859 Г. В 1863 Г. ОН СТАЛ АВТОРОМ ВСЕЙ МОТИВИРОВОЧНОЙ ЧАСТИ ЗНАМЕНИТОГО ЦИРКУЛЯРА МИНИСТРА ВНУТРЕННИХ ДЕЛ П. ВАЛУЕВА О ЗАПРЕТЕ НА ПУБЛИКАЦИИ ДЕШЕВЫХ УКРАИНСКИХ КНИГ ДЛЯ НАРОДА, В ТОМ ЧИСЛЕ ИЗВЕСТНОЙ ФОРМУЛЫ «УКРАИНСКОГО ЯЗЫКА НЕ БЫЛО, НЕТ И БЫТЬ НЕ МОЖЕТ». БИОГРАФИЧЕСКИЙ ПОДХОД К ЭТОЙ ТЕМЕ, КАК МНЕ КАЖЕТСЯ, ИМЕЕТ ОГРОМНЫЙ ПОТЕНЦИАЛ. ТАК ИЛИ ИНАЧЕ, ХОТЕЛОСЬ БЫ В БУДУЩЕМ УВИДЕТЬ КАКУЮ-ТО БОЛЕЕ СЛОЖНУЮ КАРТИНУ ЭТОЙ ГРУППЫ, ЧЕМ ТОТ ОТЧАСТИ ОДНОМЕРНЫЙ ОБРАЗ ЖЕРТВЫ ИМПЕРСКОЙ ПОЛИТИКИ И ЭГОИЗМА БОЛЕЕ БОГАТОЙ ШЛЯХТЫ, КОТОРЫЙ ДАЕТ БОВУА. 
БОВУА СОВЕРШЕННО СПРАВЕДЛИВО ОТМЕТИЛ В НЕДАВНЕМ ИНТЕРВЬЮ, ЧТО «В ДЕЙСТВИТЕЛЬНОСТИ УКРАИНСКАЯ ИДЕНТИЧНОСТЬ ФОРМИРУЕТСЯ В НАЧАЛЕ ХХ В., ПОСЛЕ РЕВОЛЮЦИИ 1905 ГОДА $\aleph^{4}$. Я БЫ УТОЧНИЛ, ЧТО ЭТОТ ПРОЦЕСС ДАЖЕ В НАЧАЛЕ ХХ ВЕКА БЫЛ ВЕСЬМА ДАЛЕК ОТ СВОЕГО ЗАВЕРШЕНИЯ, И ЕГО ВАЖНЫМИ ПОСЛЕДУЮЩИМИ ЭТАПАМИ БЫЛИ ПЕРВАЯ МИРОВАЯ ВОЙНА, ОККУПАЦИОННАЯ ПОЛИТИКА ЦЕНТРАЛЬНЫХ ДЕРЖАВ, РЕВОЛЮЦИЯ, А ЗАТЕМ СОВЕТСКАЯ ПОЛИТИКА КОРЕНИЗАЦИИ. МЕЖДУ ТЕМ В КНИГЕ КРЕСТЬЯНСТВО ПРАВОБЕРЕЖНОЙ УКРАИНЫ БОВУА НЕИЗМЕННО НАЗЫВАЕТ УКРАИНСКИМ. В ОПРЕДЕЛЕННОЙ КОНВЕНЦИИ ЭТО ДОПУСТИМО. НО НИКАКИХ ОГОВОРОК АВТОР НЕ ДЕЛАЕТ, И ВЕСЬ СЛОЖНЫЙ ПРОЦЕСС ФОРМИРОВАНИЯ НАЦИОНАЛЬНОЙ ИДЕНТИЧНОСТИ В ЭТОЙ СРЕДЕ, ВСЕ СОРЕВНОВАНИЕ УКРАИНСКОЙ, МАЛОРУССКОЙ И ОБЩЕРУССКОЙ ОРИЕНТАЦИЯ ОСТАЮТСЯ ПРАКТИЧЕСКИ БЕЗ ВНИМАНИЯ. МЕЖДУ ТЕМ ИМЕННО НА ВОЛЫНИ СОЮЗ РУССКОГО НАРОДА ИМЕЛ НАКАНУНЕ ВОЙНЫ ОКОЛО 200 ТЫСЯЧ ЧЛЕНОВ. ВООБЩЕ, КАК ЭТО СВОЙСТВЕННО МНОГИМ КНИГАМ СПЕЦИАЛИСТОВ ПО ХІХ В., ЗАЯВЛЯЮЩИХ В КАЧЕСТВЕ КОНЕЧНОЙ ХРОНОЛОГИЧЕСКОЙ ВЕХИ 1914 Г., НАЧАЛО ХХ В. В «ГОРДИЕВОМ УЗЛЕ» ВЫГЛЯДИТ СКОРЕЕ «ДОВЕСКОМ». ПОЖАЛУЙ, ТЕНДЕНЦИЯ ПОСЛЕДНЕГО ВРЕМЕНИ, КОГДА ВСЕ ЧАЩЕ В КАЧЕСТВЕ АНАЛИЗИРУЕМОГО ПЕРИОДА БЕРУТСЯ 1880-1920-Е ГОДЫ, ПОЗВОЛЯЕТ ЛУЧШЕ ПОНЯТЬ ДИНАМИКУ СОЦИАЛЬНЫХ И ПОЛИТИЧЕСКИХ ПРОЦЕССОВ В РЕГИОНЕ.

11 В ЗАКЛЮЧЕНИИ СВОЕЙ КНИГИ БОВУА ГОВОРИТ: «ЧИТАТЕЛЬ МОГ ЗАМЕТИТЬ, ЧТО Я ЗАЧАСТУЮ СМЕШИВАЮ ИСТОРИЮ С ЭТИКОЙ, ЖИВО РЕАГИРУЮ НА ИЗЛАГАЕМЫЙ МАТЕРИАЛ, ВОЗМУЩАЮСЬ, ИРОНИЗИРУЮ ИЛИ СМЕЮСЬ, ОСУЖДАЮ ИЛИ ОДОБРЯЮ» (с. 938) Я БЫ СКАЗАЛ, ЧТО ТРУДНО НЕ ЗАМЕТИТЬ ЭТОЙ ОСОБЕННОСТИ АВТОРСКОГО ОТНОШЕНИЯ К ИСТОРИИ. БЛАГОЖЕЛАТЕЛЬНО НАСТРОЕННЫЙ ЧИТАТЕЛЬ СНИСХОДИТЕЛЬНО ОТНЕСЕТСЯ К КАТЕГОРИЧНОСТИ, НЕРЕДКОЙ УПРОЩЕННОСТИ ЭТИЧЕСКИХ СУЖДЕНИЙ И ОЦЕНОК БОВУА. ТАКОЙ ЧИТАТЕЛЬ СОСРЕДОТОЧИТСЯ НА ЦЕННОМ, ЧТО ЕСТЬ В ИССЛЕДОВАНИИ. К СОЖАЛЕНИЮ, ВРЯД ЛИ ТЕ В ПОЛЬШЕ И РОССИИ, КТО СКЛОНЕН К ИДЕАЛИЗАЦИИ РОЛИ «СОБСТВЕННОЙ» СТОРОНЫ В ИСТОРИИ «КРЕСОВ» ИЛИ «ЗАПАДНЫХ ОКРАИН», И НА КОГО, СОБСТВЕННО, И СТРЕМИЛСЯ ВОЗДЕЙСТВОВАТЬ АВТОР СВОИМИ МОРАЛЬНЫМИ ОЦЕНКАМИ, ПОСЛЕДУЮТ ЭТИМ ПУТЕМ. СКОРЕЕ, ОНИ ЛИШЬ ОБРАДУЮТСЯ ВОЗМОЖНОСТИ ОТБРОСИТЬ ЭТИ ОЦЕНКИ БОВУА СО ССЫЛКОЙ НА ИХ ЭМОЦИОНАЛЬНОСТЬ, ОДНОСТОРОННОСТЬ И ОШИБКИ В АРГУМЕНТАЦИИ.

ВСЕ ЭТИ ЗАМЕЧАНИЯ И ПОЖЕЛАНИЯ, БЕЗУСЛОВНО, НЕ ОТМЕНЯЮТ ТОГО ФАКТА, ЧТО КНИГА ДАНИЭЛЯ БОВУА, ОСНОВАННАЯ НА МНОГОЛЕТНИХ ТЩАТЕЛЬНЫХ ИССЛЕДОВАНИЯХ, ЯВЛЯЕТСЯ ВАЖНЫМ ВКЛАДОМ В ОСМЫСЛЕНИЕ ПРОШЛОГО РЕГИОНА, ИСТОРИОГРАФИЯ КОТОРОГО ПО ПРЕЖНЕМУ ОСТАЕТСЯ СИЛЬНО ПОДВЕРЖЕННОЙ НАЦИОНАЛЬНЫМ ПРИСТРАСТИЯМ.

\section{NOTES}

1. D. Beauvois, Trójkąt Ukraiński - Szlachta, carat i lud na Wołyniu, Podolu i Kijowszczyźnie 1793-1914, Lublin: Wydawnictwo Uniwersytetu Marii Curie-Skłodowskiej, 2005. 
2. НА СТР. 354, НАПРИМЕР, АВТОР ГОВОРИТ О ПРАВИТЕЛЬСТВЕННЫХ МЕРАХ 1862 И 1863 ГОДОВ, ДАЖЕ НЕ НАПОМНИВ ЧИТАТЕЛЮ, ЧТО МЕЖДУ НИМИ ЛЕЖИТ РУБЕЖ НАЧАЛА ВОССТАНИЯ.

3. ПОКА МОЖНО ЛИШЬ КОНСТАТИРОВАТЬ ВАЖНЫЕ ДОСТИЖЕНИЯ УКРАИНСКОЙ ИСТОРИОГРАФИИ В ИЗУЧЕНИИ ДВОРЯНСТВА ЛЕВОБЕРЕЖНОЙ УКРАИНЫ. СМ. Т.Ф.ЛИТВИНОВА «ПОМІЩИЦЬКА ПРАВДА». ДВОРЯНСТВО ЛІВОБЕРЕЖНОЇ УКРАЇНИ ТА СЕЛЯНСЬКЕ ПИТАННЯ НАПРИКІНЦІ ХVIII - В ПЕРШІЙ ПОЛОВИНІ ХІХ СТ. (ІДЕОЛОГІЧНИЙ АСПЕКТ) / Т.Ф. ЛИТВИНОВА. - ДНіПРОПЕТРОВСЬК.: ЛІРА, 2011

4. СПОР О КРЕСАХ. С ПРОФЕССОРОМ ДАНИЭЛЕМ БОВУА БЕСЕДУТ МАРТИН ВОЙЦЕХОВСКИЙ // НОВАЯ ПОЛЬША. №7-8, 2012. http://www.novpol.ru/index.php?id=1709 